\title{
Epidemiological Survey of Extrapulmonary Tuberculosis in Baghmalek City of Khuzestan Province in Iran (2007-2011)
}

\author{
Masoumeh Yahyavi ${ }^{1, *}$; Amin Delavari ${ }^{1}$; Zahra Karimi ${ }^{2}$; Sajad Falsafi-Zadeh ${ }^{2}$; Shahla \\ Samieifar ${ }^{1}$; Fariba Abbasi ${ }^{3}$; Mitra Moradi ${ }^{3}$ \\ ${ }_{1}^{1}$ Department of Laboratory Science, School of Paramedicine, Ahvaz Jundishapur University of Medical Sciences, Ahvaz, IR Iran \\ ${ }_{3}^{2}$ Department of Biology, Faculty of Science, Shahid Chamran University, Ahvaz, IR Iran \\ ${ }^{3}$ Golestan Hospital, Ahvaz Jundishapur University of Medical Sciences, Ahvaz, IR Iran \\ ${ }^{*}$ Corresponding author: Masoumeh Yahyavi, Department of Laboratory Science, School of Paramedicine, Ahvaz Jundishapur University of Medical Sciences, Ahvaz, IR Iran. Tel: \\ +98-6133386171, E-mail: myehyavi@yahoo.com
}

Received: January 5, 2015; Accepted: March 25, 2015

\begin{abstract}
Background: Mycobacterium tuberculosis is still an important cause of pulmonary and extrapulmonary tuberculosis (TB) and a major cause of death in the world. Extrapulmonary TB is spread almost as much as one third of pulmonary TB.

Objectives: The aim of this study was to investigate the prevalence of $\mathrm{TB}$ and type of affected tissue in patients with extrapulmonary TB in Tuberculosis Center in Baghmalek City.

Patients and Methods: This retrospective cross-sectional study was performed on the medical records of patients with TB referred to Tuberculosis Center in Baghmalek City over a five-year period. Data included information about age, sex, location, and affected tissue or organ.

Results: Among 145 patients with TB, 67 patients (12.25\%) were diagnosed with extrapulmonary TB including 39 males and 28 females with the age range of 15 to 64 years. Most common organ involved in the extrapulmonary TB was the lymph nodes (29\%) followed by TB meningitis (17\%), urinary tract TB (17\% each), bone TB (13\%), and pleural TB (13\% each).

Conclusions: The findings suggest that the prevalence of extrapulmonary TB was significant. Therefore, it is necessary to perform tests for extrapulmonary TB besides tests for TB.
\end{abstract}

Keywords: Tuberculosis; Bone and Bones; Lymph Nodes

\section{Background}

Tuberculosis (TB) is one of the most common infectious diseases nowadays. It has ability to involve all organs of the body but the lungs are more likely prone to develop TB. The most infected organs include lymph nodes, pleura, genitourinary system, bones, joints, and meningitis (1, 2). It is estimated that one in three people in the world, is infected with Mycobacterium tuberculosis, and one person is added to them every second. It is estimated that a significant number of patients are multidrug resistant to TB (MDR-TB) (3).

In 2010 , about 8.8 million persons were infected with active TB, and 1.1 million persons died of TB. TB is the biggest cause of death from single-factor infectious disease worldwide (even more than AIDS, malaria, and measles), and have a tenth rank in global burden of disease. It is predicted that the disease will continue to maintain its present position until 2020 or come up to seventh degree. Currently, 12 million persons are infected with TB worldwide more than of $80 \%$ of which are in 22 developing countries (4-6).

Diagnosis of extrapulmonary TB is often difficult be- cause of uncertain clinical symptoms and the need for revascularization. The disease emerges with different signs in different situations; therefore, it is known as "thousand faces disease" (2). Delay in diagnosis and treatment of pulmonary and extrapulmonary TB is an important factor in spreading and developing symptom of disease. Medical studies have shown that symptoms of TB are cough, weight loss, fatigue, fever, and night sweats in half of the cases (1-3). Extrapulmonary TB has the reverse epidemiologic trend of pulmonary TB. Over the last several years, reported cases of extrapulmonary TB had an increasing trend in absolute numbers and proportion of all reported TB cases.

Prevalence of pulmonary TB in Iran in 2011 was 14.6 per 100000 persons of which 4.12 had extrapulmonary TB. Among the provinces, Sistan and Baluchestan and Golestan showed the highest incidence in the country (6). The reported rates of pulmonary TB in different areas are different. According to Centers of America diseases report, incidence of disease was $18 \%$ in 2010 . The incidence of extrapulmonary TB has been reported to be $22.5 \%$ in 2010

Copyright (C) 2015, Ahvaz Jundishapur University of Medical Sciences. This is an open-access article distributed under the terms of the Creative Commons Attribution-NonCommercial 4.0 International License (http://creativecommons.org/licenses/by-nc/4.0/) which permits copy and redistribute the material just in noncommercial usages, provided the original work is properly cited. 
according to report of National Research Institute of Tuberculosis, and the most infected organ was lymph node. The incidence of extrapulmonary TB in Chaharmahal and Bakhtiari province, Tabriz city, and Khorasan province were reported to be $27 \%, 41 \%$, and $33.5 \%$, respectively (5-10). Since Iran is an endemic area of TB and TB has different clinical signs depending on demographic changes over time, it is necessary to investigate prevalence and clinical findings of pulmonary and extrapulmonary TB in different areas of country.

\section{Objectives}

Therefore, the purpose of this study was to investigate epidemiology of extrapulmonary TB in Baghmalek City, which is located in the east of Khuzestan province.

\section{Patients and Methods}

This retrospective cross-sectional study was performed at Tuberculosis Diagnosis Laboratory of Health Center of Baghmalek City from 2007 to 2011. Baghmalek City is located in east of Khuzestan Province, southwest of Iran, and near Chaharmahal and Bakhtiari Province and Kohgiluyeh and Boyer-Ahmad Province. Its population is about 20844. In this study, of 145 patients with TB, 67 patients with extrapulmonary TB were studied. All reports of tissue samples to diagnose necrotizing granuloma of TB or non-TB infectious were extracted from the archive section. Then non-pulmonary tissues were separated and relevant histopathologic sections were reinvestigated. The slides were stained by Hematoxylin \& Eosin and Ziehl-Neelsen staining. Mycobacterial infectious was confirmed by the observation of acid-fast bacilli (2-4). Extracted variables from patients' records and standardized tests included individual variables such as age, sex, organ involvement, residence status, and nationality. All the information had been recorded in the pre-designed checklist and description analysis and distribution of related variables were performed by SPSS 19 (SPSS Inc., Chicago, Illinois, the United States).

\subsection{Ethical Considerations}

This study was conducted after obtaining the approval of the Ethics Committee of Ahvaz Jundishapur University of Medical Sciences.

\section{Results}

Out of 350 patients with suspicious extrapulmonary TB, 67 (19.14\%) had extrapulmonary TB. Among 145 patients with $\mathrm{TB}, 78$ patients (53.79\%) had pulmonary TB, and 67 (46.20\%) had extrapulmonary TB. Thirty-nine patients with extrapulmonary TB were male and 28 were female (Table 1). Lymph nodes were the most common involved organ (20 cases; $29.85 \%$ ). TB Meningitis with prevalence rate of $17.91 \%$ was the second most prevalent involved organ. Pleural and bones TB showed less prevalence in com- parison to lymph node TB and TB Meningitis. Extrapulmonary TB such as gastrointestinal tract, uterus, visceral, renal, breast, and pericardium TB have shown lower incidence rates among patients. Most patients with extrapulmonary TB were at two age groups: 15 to 24 and 45 to 54 years old, with incidence of $41.79 \%$ and $23.88 \%$, respectively (Table 2). In addition, $61.19 \%$ of patients inhabited in rural area and $38.81 \%$ in urban area (Table 1). Nationality of all patients was Iranian.

Table 1. Distribution of Individual Variables in Patients With Extrapulmonary Tuberculosis in Health Center of Baghmalek City

\begin{tabular}{lc}
\hline Variables & Frequency (\%) \\
\hline Residency status & \\
Urban & $26(38.81)$ \\
Rural & $41(61.19)$ \\
Gender & \\
Male & $39(58.20)$ \\
Female & $28(41.79)$ \\
\hline
\end{tabular}

Table 2. The Age Distribution of Patients With Extrapulmonary Tuberculosis in Health Center of Baghmalek City

\begin{tabular}{lc}
\hline Age, $\mathbf{y}$ & Frequency (\%) \\
\hline$<\mathbf{1 5}$ & $0(0)$ \\
$\mathbf{1 5 - 2 4}$ & $28(41.79)$ \\
$\mathbf{2 5 - 3 4}$ & $10(14.92)$ \\
$\mathbf{3 5 - 4 4}$ & $6(8.95)$ \\
$\mathbf{4 5 - 5 4}$ & $16(23.88)$ \\
$\mathbf{5 5 - 6 4}$ & $7(10.44)$ \\
$>\mathbf{6 4}$ & $0(0)$ \\
\hline
\end{tabular}

\section{Discussion}

Extrapulmonary TB is one of the highly prevalent diseases in developing countries including Iran. In this study, the prevalence of extrapulmonary TB infection was $19.14 \%$, which was greater than the incidence reported in Nigeria (5\%) and India (3.9\%), but lower than the prevalence observed in Turkey (25.9\%) and Southern region of Ethiopia (28\%) (9-13). The difference between the current study and previous reports on extrapulmonary TB might be the result of variation in diagnostic methods; the majority of the previous reports were done by culture, cytological studies, and/or pericardoscopy. Nevertheless, extrapulmonary TB remains a disease of major importance in public health and it has been increasing from time to time.

The results of this study showed higher prevalence of pulmonary TB in males than in females, while extrapulmonary TB was more prevalent in females. In this regard, 
Yahyavi M et al.

results of studies by Mousavi et al. (11) and Rokni et al. (9) were consistent with our results. Men are more susceptible to TB because of the type of employment and smoking. Comparison between incidence of pulmonary and extrapulmonary TB in Kashan City by Mousavi et al. (11) as well as the study of Aminzadeh et al. (10) showed that the prevalence of pulmonary TB was more than that of extrapulmonary TB. However, extrapulmonary TB prevalence rate is increasing rapidly (Table 1 ). The probable reasons for increasing trend of extrapulmonary TB might be high TB burden, underdiagnosis of pulmonary TB, or transmission of other mycobacteria (10-15).

In this study, the most infected organ was lymph glands (29.85\%). Incidence of lymph node TB was $23.2 \%$ in study of Metanat et al. (15) but study of Aminzadeh et al. (10) in Tehran (35.8\%) and Rokni et al. (9) in Mashhad (33.3\%) showed more incidence in comparison to our study. A study in Hong Kong showed that pleural TB was the most prevalent extrapulmonary TB followed by lymph node TB (Figure 1) (13).

The prevalence of TB meningitis was $17.91 \%$ and stood at second rank among other organs involved with TB. In Rokni et al. (9) and Valizadeh et al. (14) studies, the incidence rate of TB meningitis was similar to ours (9-14). However, Mousavi et al. (11), Metanat et al. (15), and Mardani et al. (16) studies show bones TB as the second most prevalent extrapulmonary TB after lymph node TB.

Incidence of bone TB was 13.43 in our study. The prevalence of bone TB in Metanat et al. (15), Mardani et al. (16), Rokni et al. (9), and Aminzadeh et al. (10) study was reported to be $12 \%, 18 \%, 11.5 \%$, and $14.8 \%$ respectively(Figure 1 ). Pleural TB with $13.43 \%$ incidence showed equal incidence with bone TB (Figure 1). Therefore, these two organs are listed as the third most common involved organs in extrapulmonary TB. Prevalence of pleural TB has been reported $12.2 \%$ and $20.7 \%$ in Metanat et al. (15) and Rokni et al. (9) studies, respectively $(9,15)$. A study in Shahryar City has reported prevalence for plural TB to be $9.8 \%(14)$.

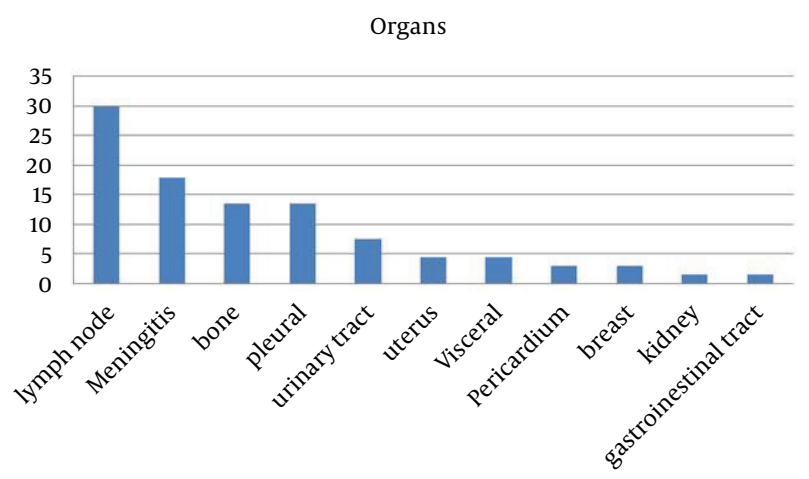

Figure 1. Frequency of Involved Organs in Extrapulmonary Tuberculosis in Patients of Health Center of Baghmalek City
Metanat et al. (15) study is consistent with our study in prevalence of gastrointestinal tract TB (1.49\%). However, the incidence of gastrointestinal tract TB was reported to be $9.8 \%$ in Valizadeh et al. (14) study (Figure 1). Gastrointestinal TB symptoms include pain, loss of appetite, diarrhea, intestinal obstruction, ascites, and bleeding. In this study, the number of patients living in rural areas was more than the number of patients residing in the city. The most prevalent age groups were 15 to 24 years old group. Therefore, TB elimination strategies should include targeted evaluation and appropriate treatment of individuals in high-prevalence groups $(11,13)$.

Due to the long duration of beginning illness before admission of patients in hospital and primary diagnosis, examination of other organs to identify final pathologic diagnosis is necessary. TB must be considered as a disease with diverse clinical symptoms. Since the number of patients with TB in rural population is high, it is necessary to expand health-therapy services for rural health centers and training people.

Awareness of such regional differences in the epidemiology of TB will be very helpful to physicians and other healthcare providers involved in the diagnosis, treatment, and prevention of TB. Screening of lymph node and other body fluid specimens for extrapulmonary TB could help for treatment, control, and prevention of the disease. The results suggest that, besides pulmonary TB, emphasis should be placed on laboratory diagnosis and treatment of extrapulmonary TB.

\section{Acknowledgements}

We would gratefully acknowledge the study participants who provided us the histopathological specimens and laboratory staff working in the Pathology Unit of the Health Center of Baghmalek city who kindly supported us during study.

\section{Authors' Contributions}

All authors contributed extensively to the work presented in this paper.

\section{Funding/support}

Ahvaz Jundishapur University of Medical Sciences supported the study financially.

\section{References}

1. Haas DW. Mycobacterial diseases. In: Mandell GL, Bennet JE, Dolin R editors. Principles and Practice of Infectious Disease.. Philadelphia, PA: Churchill Livingstone; 2000. pp. 2576-25607.

2. Hussein MM, Mooij JM, Roujouleh H. Tuberculosis and chronic renal disease. Semin Dial. 2003;16(1):38-44.

3. Mahon CR, Lehman D, Manuselis G. Textbook of diagnostic microbiology:: Elsevier Health Sciences; 2014.

4. World Health Organization.. Global Tuberculosis Report. Geneva, Switzerland: WHO; 2012. Available from: http:/|apps.who.int/iris/ bitstream/10665/75938/1/9789241564502_eng.pdf.

5. Centers for Disease Control and Prevention.. Reported Tuberculo- 


\section{Yahyavi M et al.}

sis in the United States. 2011. Available from: http://www.cdc.gov/ tb/statistics/reports/2011.

6. Iranian Center for the Control and Prevention of Diseases.. Epidemiological situation of tuberculosis in Iran.: Ministry of Health; 2011.

7. Yahyavi M, Karimi Z, Falsafi-zadeh S, Noori M, Hosinzadeh F, Moradi F. Epidemiologic consideration of Tuberculosis in East Health Center of Ahvaz in 2010. Jundishapour J Chronic Dis Care. 2014;3(1):41-5.

8. Khatib S. [Evaluation of extrapulmonary tuberculosis in Chahar Mahal and Bakhtiari province]. J Shahrekord Univ Med Sci. 1998;3(1):35-40.

9. Rokni F, Etemadi J. [Epidemiology of extra pulmonary tuberculosis in the city of Mashhad]. Med J Mashhad Univ Med Sci. 2003;87(1):9-23.

10. Aminzadeh Z, Akhyany H. [Clinical manifestations of Patients with extra-pulmonary tuberculosis in Loghman Hospital of Tehran]. Tabib shargh. 2006;7(4):283-7.
11. Mousavi GA, Saberi H, Sharif A, Ghorbani F, Shadkam M, Vojdani $S$, et al. A comparative study of patients with pulmonary tuberculosis and extrapulmonary tuberculosis in Kashan during 2002-7. J Kashan Univ Med Sci. 2009;13(3):235-41.

12. Farazi A, Sofian M, Jabbari asl M. Extra-pulmonary tuberculosis and its comparison with pulmonary tuberculosis in Markazi province (2002-2012). Arak Med Univ J. 2013;16(74):58-66.

13. Noertjojo K, Tam CM, Chan SL, Chan-Yeung MM. Extra-pulmonary and pulmonary tuberculosis in Hong Kong. Int J Tuberc Lung Dis. 2002;6(10):879-86.

14. Valizadeh S, Memariani M, BegVerdi R, Memariani H. [Epidemiology of extra-pulmonary tuberculosis in Shahryar city in the year 2008].J Med Microbiol. 2010;3(1):55-8.

15. Metanat M, Salehi M, Sharifimood B, Jahantigh AR, Rohani Z. [Epidemiology of extra pulmonary tuberculosis in Zahedan]. Tabib shargh. 2006;7(4):275-80.

16. Mardani M. [Extrapulmonary tuberculosis and review of 146 cases].J Med Counc I.R. Iran. 2008;18(4):282-7. 